\title{
Qualidade de vida de crianças ostomizadas na ótica das crianças e das mães
}

\author{
Quality of life of children with stomas: the children and mothers' point of view
}

\author{
Simone G. Barreire ${ }^{1}$, Olcinei A. Oliveira ${ }^{1}$, Wilma Kazama ${ }^{1}$, \\ Miako Kimura $^{2}$, Vera L.C.G. Santos ${ }^{3}$
}

\section{Resumo}

Objetivos: analisar a qualidade de vida de crianças ostomizadas em sua ótica e na de suas mães; comparar os escores de qualidade de vida (QV) obtidos em ambos grupos (crianças e mães), e verificar as associações entre os escores de qualidade de vida e as características sociodemográficas e clínicas das crianças.

Métodos: após o cumprimento das exigências éticas, os dados sobre a qualidade de vida foram obtidos através do Autoquestionnaire Qualité de Vie Enfant Imagé (AUQEI), em sua versão traduzida para a língua portuguesa, adaptada por Assumpção et al. e validada para este estudo. O questionário foi respondido por 20 crianças ostomizadas, oriundas de um serviço público infantil de referência do Município de São Paulo e por suas respectivas mães. Os dados obtidos foram submetidos ao teste de normalidade de KolmogorovSmirnov, coeficientes de correlação ordinal de Spearman e de correlação linear de Pearson, e o teste $t$ de Student.

Resultados: dez meninos e 10 meninas, com idades entre 4 e 12 anos, apresentaram idade média de 8,7 anos $(\mathrm{DP}=2,64)$, com predomínio da raça branca $(55 \%)$ e da escolaridade fundamental $(65 \%)$. Clinicamente, predominaram os estomas urinários $(55 \%)$, provenientes, principalmente, das malformações congênitas $(60 \%)$. Com relação à qualidade de vida, obtiveram-se escores médios totais de 51,95 (DP = 7,90) e 49,60 (DP = 5,60), apontados, respectivamente, pelas crianças e mães. Quanto aos fatores, os escores obtidos pelas crianças foram 8,$14 ; 8,29 ; 10,27$ e 11,41 , respectivamente, para autonomia, lazer, funções e família. Correlações estatisticamente significativas foram verificadas entre a escolaridade e o EQVMãe $(r=-0,56)$ e com o EQVCr $(r=-0,63)$ e entre o EQVMãe e os Fatores Família $(\mathrm{r}=0,86)$, Autonomia $(\mathrm{r}=0,60)$ e Funções $(\mathrm{r}=0,59)$.

Conclusões: este estudo vem contribuir para a melhor compreensão sobre a qualidade de vida de crianças com ostomias, bem como alguns desses aspectos na ótica das mães.

J Pediatr (Rio J) 2003;79(1):55-62: estomaterapia, estomas pediátricos, qualidade de vida, AUQEI.

1. Enfermeiro estomaterapeuta, Escola de Enfermagem da USP

2. Professor Livre Docente, Escola de Enfermagem da USP.

3. Professor Doutor, Escola de Enfermagem da USP.

Artigo submetido em 29.05.02, aceito em 13.11.02

\begin{abstract}
Objectives: to analyze the quality of life of children with stomas according to the children and mothers' point of view; to compare the results related to quality of life in both groups, and to verify the statistical associations between those aspects and children's demographic and clinical data.
\end{abstract}

Methods: after the fulfilling ethical requirements, the specific data were obtained using the Autoquestionnaire Qualité de Vie Enfant Imagé, adapted and validated for Brazilian culture and also for this study by Assumpção Jr. et al.. It is composed by 26 questions, 18 of them are distributed among four factors: Functions, Family, Autonomy and Leisure.

Results: twenty children, aged 4 to 12 years, from a Specialized Outpatient Ostomy Care Center for Children and their respective mothers answered the questionnaire. Ten boys and 10 girls participated, their mean age was $8.7(\mathrm{SD}=2.64)$ years, with the predominance of Caucasians (55.0\%), and elementary education level $(65.0 \%)$. Clinical profile shows a predominance of urinary stomas $(55.0 \%)$ caused by congenital diseases $(60.0 \%)$. Related to quality of life, the total mean scores were $51.95(\mathrm{SD}=7.90)$ and 49.60 ( $\mathrm{SD}=5.60)$, respectively for children and mothers, without statistically significant difference. Regarding each factor, the patients' scores were 8.14, 8.29, 10.27 and 11.41 , respectively for autonomy, leisure, functions and family. Some statistically significant associations were verified between education level and mothers' quality of life $(r=-0.56)$ and children's quality of life $(r=-0.63)$, and also between mothers' quality of life and family $(r=0.86)$, autonomy $(r=0.60)$ and functions $(r=0.59)$.

Conclusions: This study improves the comprehension about quality of life of children with stoma as well as some of these aspects on their mothers' point of view.

JPediatr (Rio J) 2003;79(1):55-62: ostomy care, stomal therapy, pediatric stomas, quality of life, AUQEI.

As ostomias constituem uma medida terapêutica cirúrgica de caráter provisório ou definitivo, para muitas doenças ou situações clínicas ${ }^{1}$, também entre as crianças. Para essas, decorrem principalmente de malformações congênitas, obstruções intestinais e traumas ${ }^{2-3}$. 
Dentre os estomas digestivos na infância, as colostomias são freqüentemente realizadas e, em sua maioria, ocorrem no período neonatal, objetivando descomprimir, proteger anastomoses, ou restaurar a função do órgão afetado, quando é impossibilitada a sutura primária ${ }^{2-3}$. Quanto ao sistema geniturinário, predominam as malformações congênitas, porém a criança está ainda sujeita a traumas abdominais decorrentes de acidentes automobilísticos, ou mesmo domésticos. Dentre os estomas urinários, têm-se as nefrostomias, ureterostomias, cistostomias e vesicostomi$\mathrm{as}^{3}$. Os estomas em pediatria são primordialmente temporários, e a reconstrução do trânsito gastrintestinal, ou urinário depende da doença de base e das intervenções cirúrgicas necessárias.

A falta de controle esfincteriano causa, nas pessoas de todas as idades, muitos conflitos e sentimentos de rejeição, de culpa e ansiedade, permeados pelos distúrbios de imagem corporal e identidade. Configuram-se alterações não somente de ordem física e de autocuidado, mas também psicológicas, sociais e espirituais, provavelmente também para as crianças e, particularmente, para seus familiares ou responsáveis mais diretos.

A criança como um ser em contínuo processo de desenvolvimento e crescimento, ao apresentar alterações físicas, corporais ou comportamentais, além da hospitalização, que acarretam limites de oportunidades para vivenciar situações que lhe permitam agir e descobrir o mundo, pode ter sua personalidade violentada através da perda de segurança e dano ao próprio desenvolvimento, refletindo, conseqüentemente, na sua vida adulta ${ }^{4}$.

Durante cada fase do desenvolvimento da criança, qualquer condição que ameace a integridade corporal ou a autoimagem será percebida de maneira diferente e demandará mecanismos de defesa e adaptativos específicos para a faixa etária. De qualquer maneira, em todas essas etapas do desenvolvimento físico e psicoemocional, o binômio criança-família deve constituir o foco de atenção do profissional de saúde, particularmente quando ocorre a mutilação neste caso, através da formação de um estoma - seja no nascimento, como é bastante freqüente, seja mais tardiamente. Quanto mais precoce for a abordagem de pais e crianças a respeito da doença, maiores chances de estabelecimento de um vínculo de confiança, otimizando o tratamento clínico e contribuindo para um crescimento mais saudável ${ }^{5-9}$. Dessa maneira, também para essa clientela impõe-se estabelecer a melhoria da qualidade de vida $(\mathrm{QV})$ como meta do processo de cuidar.

Sobre as definições de QV, pode-se afirmar que elas são tão numerosas como os métodos de avaliá-las e que não existe consenso sobre o seu significado. Na literatura científica, observam-se inúmeras controvérsias teóricas e metodológicas, principalmente em função do caráter multidimensional e subjetivo do conceito, mas também pelos inúmeros fatores que interferem na sua construção como o objeto de avaliação ou de pesquisa ${ }^{10-11}$. QV é um termo representado pela tentativa de nomear algumas características da experiência humana, na perspectiva do próprio indivíduo através de sua percepção subjetiva (Guyatt), e tem, como fator central, a sensação de bem-estar, conforme alguns autores ${ }^{12-14}$.

Segundo Hinds, mencionado por Assumpção Jr et al. ${ }^{13}$, para crianças e adolescentes, bem-estar pode significar “...o quanto seus desejos e esperanças se aproximam do que realmente está acontecendo. Também reflete sua prospecção, tanto para si quanto para os outros..." e é sujeita a alterações, sendo influenciada por eventos cotidianos e problemas crônicos. Assumpção Jr et al. ${ }^{13}$ também afirmam que QV é um conceito central, que determina a sensação subjetiva de bem-estar, também para as crianças, e que estas são e sempre foram capazes de se expressar quanto a essa subjetividade.

Nesse sentido, Eiser, Pantell, Lewis e Canning apud Assumpção Jr et al. ${ }^{13}$ atentam para a diferença crucial que existe entre o que é qualidade de vida infantil na visão de um adulto e da própria criança. Para os autores, as percepções dos pais e da equipe médica em contato com a criança sob avaliação apresentam baixos índices de correlação com a auto-avaliação infantil. Por outro lado, Magnificat e Dazord $^{15}$, ao reportarem-se à intrínseca dependência física, psíquica e jurídica das crianças em relação aos seus pais, especialmente quando mais jovens, afirmam que ocorrem íntimas e múltiplas relações entre a QV dos pais e crianças, exigindo-se avaliações paralelas entre ambos.

No que tange especificamente à QV da criança ostomizada, pouco se conhece ${ }^{16-20}$, havendo muitas especulações e pontos de vista variados. Enquanto alguns sugerem que o estoma é devastador para a imagem corporal na infância e na adolescência, outros acreditam que a doença é a causa. Por isso, somente resultados de pesquisas metodologicamente bem conduzidas podem suplantar tais especulações e, talvez, oferecer sugestões para programas e políticas assistenciais pautados nesses achados, em busca da reabilitação dessas crianças e de seus familiares. Essas considerações motivaram a realização deste estudo, objetivando avaliar a QV dessa clientela, na ótica das próprias crianças e de suas mães, e verificar as associações existentes entre esses resultados e as características demográficas e clínicas das crianças.

\section{Casuística e métodos}

Este estudo, de caráter descritivo, exploratório, transversal e com abordagem quantitativa, foi realizado em um hospital público infantil da cidade de São Paulo, único de referência para o atendimento interdisciplinar de crianças ostomizadas provenientes de serviços da Grande São Paulo e do Interior do estado.

Sessenta e três crianças, na faixa etária de 4 a 12 anos, estavam cadastradas no serviço à época da coleta de dados. Dessas, 20 (31,7\%) crianças e respectivas mães compare- 
ceram para entrevista, conforme agendamento prévio, compondo a amostra deste estudo. Além da faixa etária, as crianças deveriam atender os seguintes critérios de inclusão: apresentar condições mentais, intelectuais e de comunicação para serem entrevistadas; e ter autorização das mães para participar do estudo.

Após cumprimento das exigências éticas, a coleta de dados foi precedida de informação sobre os objetivos e procedimentos da pesquisa junto às mães das crianças, as quais eram, então, solicitadas a assinarem os termos de consentimento pós-informado em que autorizavam a participação da criança e da sua própria no estudo. A partir disso, passava-se à entrevista, em separado, das crianças e das mães, as quais, no entanto, permaneciam junto aos filhos durante todo o procedimento.

Os dados foram obtidos utilizando-se um instrumento que continha duas partes: a primeira para a obtenção dos dados demográficos e clínicos das crianças ostomizadas (levantados dos prontuários), e a segunda para a avaliação da qualidade de vida através da Escala de Qualidade de Vida da Criança (Figura 1), versão adaptada para o português por Assumpção Jr. et al ${ }^{13}$.

A EQVC ou Autoquestionnaire Qualité de Vie Enfant Imagé (AUQEI) foi desenvolvida por Manificat e Dazord $^{15}$, em 1997, em estudo com 95 crianças, das quais $60 \%$ sem problemas de saúde, $26 \%$ pós-transplante renal e $14 \%$ soropositivas para HIV, com idades entre 3 anos e 9 meses a 12 anos e 6 meses. Sua versão atual é composta de 26 questões ou domínios que exploram relações familiares, sociais, atividades, saúde, funções corporais e separação, 18 deles contidos em 4 fatores ou dimensões, assim constituídos:

- função: questões relativas às atividades na escola, às refeições, ao deitar-se, e à ida a médico (questões $1 ; 2 ; 4 ; 5 ; 8)$;

- família: questões relativas à opinião quanto às figuras parentais e delas, quanto a si mesmo (questões $3 ; 10 ; 13 ; 16 ; 18)$;

- lazer: questões relativas a férias, aniversário e relações com os avós (questões 11;21;25);

- autonomia: questões relacionadas à independência, relação com os companheiros e avaliação (questões $15 ; 17 ; 19 ; 33 ; 24)$.

A escala, adequada à especificidade e ao contexto em pediatria, incluindo a dimensão subjetiva, visa a avaliar a sensação de bem-estar, ou satisfação das crianças em relação a alguns aspectos da vida, no momento atual, sem partir de inferências realizadas sobre o seu desempenho e produtividade.

No Brasil, o instrumento foi validado por Assumpção Jr et al. ${ }^{13}$ junto a 353 crianças saudáveis pertencentes a uma escola privada do município de São Paulo, atestando suas propriedades psicométricas e obtendo uma nota de corte de 48, abaixo da qual a QV das crianças estudadas foi considerada prejudicada.
Operacionalmente, consiste de auto-avaliação feita pela criança com o suporte de quatro figuras ou faces, que expressam diferentes estados emocionais, associados a diversos domínios da vida. Inicialmente, solicita-se à criança que ela apresente uma experiência própria vivida frente a cada uma das alternativas reportadas pelas quatro imagens. Uma vez identificados os estados emocionais correspondentes, passa-se à leitura das questões, pedindo que ela assinale, sem tempo definido, a resposta que mais corresponde ao domínio proposto. Neste estudo, para as crianças com idade menor e com dificuldade de leitura, o questionário foi lido pelos pesquisadores, sem qualquer outro tipo de instrução. Os escores podem variar de 0 a 3 correspondentes, respectivamente, a muito infeliz, infeliz, feliz, muito feliz, e a escala possibilita a obtenção de um escore único, resultante da somatória dos escores atribuídos aos itens. As questões de números 6, 7, 9, 12, 14, 20, 22 e 26 não estão incluídas nos quatro fatores e detêm importância isolada, pois representam domínios separados dos demais. Para efeito de discussão dos escores totais, utilizou-se a nota de corte 48, obtida e proposta por Assumpção Jr. et al. ${ }^{13}$.

\section{Procedimentos estatísticos}

Os resultados obtidos foram submetidos aos seguintes procedimentos estatísticos: intervalos de confiança de $95 \%$, para estimar o valor médio real das variáveis quantitativas e porcentagens reais de alguns eventos de interesse; teste de normalidade de Kolmogorov-Smirnov, para testar a hipótese de normalidade dos escores observados para os domínios do instrumento; coeficientes de correlação ordinal de Spearman e de correlação linear de Pearson empregados na análise das correlações; e o test $t$ de Student, para amostras independentes, utilizado nas comparações. Pvalue inferior a 0,05 foi considerado estatisticamente significante para todas as análises.

Para a validação da EQVC para a população alvo, utilizaram-se os mesmos procedimentos adotados por Manificat, Dazord ${ }^{15}$ e por Assumpção Jr. et al. ${ }^{13}$. Assim, foram verificadas a confiabilidade, através da análise da consistência interna medida pelo coeficiente alfa de Cronbach; a validade externa medida pela correlação ordinal entre os escores de $\mathrm{QV}$, obtidos pelas crianças e respectivas mães; e a validade de construto, através da correlação interfatores, medida pela correlação ordinal entre os fatores que compõem a escala.

\section{Resultados}

Os resultados são apresentados em números absolutos e relativos, sob a forma de tabelas e gráficos, e foram distribuídos em Validação da EQVC, Caracterização demográfica e clínica das crianças ostomizadas e Análise da QV das crianças ostomizadas. 


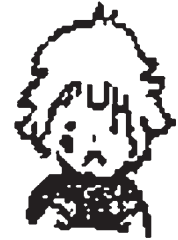

Algumas vezes você está muito muito infeliz? Diga por quê:

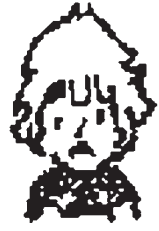

Algumas vezes você está infeliz? Diga por quê:

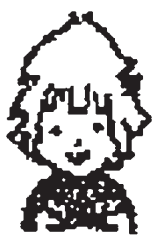

Algumas vezes você está feliz?

Diga por quê:

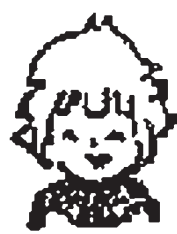

Algumas vezes você está muito feliz? Diga por quê:

\section{Diga como voce se sente:}

\section{Muito infeliz Infeliz}

Feliz Muito infeliz

1. à mesa, junto com sua família

( )

( )

( )

( )

( )

( )

6. quando você vê uma fotografia sua

7. em momentos de brincadeira, durante o recreio escolar

8. quando você vai a uma consulta médica

9. quando você pratica um esporte

10. quando você pensa em seu pai ....

11. no dia do seu aniversário

12. quando você faz as lições de casa

13. quando você pensa em sua mãe

14. quando você fica internado no hospital

15. quando você brinca sozinho(a)

16. quando seu pai ou sua mãe falam de você

17. quando você dorme fora de casa

18. quando alguém te pede que mostre alguma coisa que você sabe fazer ...

19. quando os amigos falam de você

20. quando você toma os remédios

21. durante as férias

22. quando você pensa em quando tiver crescido

23. quando você está longe de sua família

24. quando você recebe as notas da escola

25. quando você está com seus avós

26. quando você assiste televisão
( ) ( )

( )

( )

( )

( )

( )

( )

( )

( )

( )

( )

( )

( )

( )

( )

( )

( )

( )

( )

( )

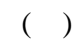

( )

( )

( )

( )

( )

( )

( )

( )

( )

( )

( )

( )

( )

( )

( )

( )

( )

( )

( )

( )

( )

( )

( )

( )
( )

( )

( )

( )

( )

( )

( )

( )

( )

( )

( )

( )

( )

( )

( )

( )

( )

( )

( )

( )

( )

( )

( )

( )

( )

( )
( )

( )

( )

( )

( )

( )

( )

( )

( )

( )

( )

( )

( )

( )

( )

( )

( )

( )

( )

( )

( )

( )

( )

( )

( )

( )

Figura 1 - AUQUEI - Questionário de avaliação de qualidade de vida em crianças e adolescentes de Magnificat e Dazord 


\section{Validação da EQVC}

A consistência interna, avaliada pelo coeficiente alfa de Cronbach, calculado para os 26 itens do instrumento, produziu um valor de 0,60 e mostrou-se satisfatória para a população deste estudo.

A validade externa foi atestada através da obtenção de forte correlação positiva e estatisticamente significativa $(\mathrm{r}=0,82 \mathrm{e} \mathrm{p}=<0,05)$ entre os escores médios totais de $\mathrm{QV}$, obtidos pelas crianças e suas mães.

Os resultados mostraram, ainda, correlações positivas, de moderada $(0,30$ a 0,50$)$ a forte intensidade $(>0,50)$, e estatisticamente significantes $(\mathrm{p}<0,05)$, entre os fatores família e lazer e família e função, sugerindo a validade de construto do instrumento utilizado.

\section{Caracterização demográfica e clínica das crianças ostomizadas}

Verifica-se, através dos dados da Tabela 1, que a amostra estudada foi constituída de 20 crianças, com média etária de 8,7 anos ( $\mathrm{DP}=2,64)$, predominando a faixa de 10 a 12 anos ( 9 crianças ou $45 \%$ ). Quanto ao sexo, a distribuição foi eqüitativa entre meninos e meninas ( 10 ou $50 \%$ para cada um) e 11 (55\%) possuíam cor branca. Com relação à

Tabela 1 - Distribuição das variáveis sociodemográficas da amostra - São Paulo, 2001

\begin{tabular}{|c|c|c|}
\hline \multirow[t]{2}{*}{ Variável } & \multicolumn{2}{|c|}{ Distribuição } \\
\hline & $\mathbf{N}^{\mathbf{o}}$ & $\%$ \\
\hline \multicolumn{3}{|l|}{ Sexo } \\
\hline Masculino & 10 & 50,0 \\
\hline Feminino & 10 & 50,0 \\
\hline IC $95 \%$ para feminino & $50,0 \pm 24,0$ & \\
\hline \multicolumn{3}{|l|}{ Idade (em anos) } \\
\hline $4 \mid-6$ & 3 & 15,0 \\
\hline $6 \mid-8$ & 4 & 20,0 \\
\hline $8 \mid-10$ & 4 & 20,0 \\
\hline $10 \mid-\mathrm{I} 12$ & 9 & 45,0 \\
\hline Média (DP) & $8,7(2,64)$ & Mediana: 9,0 \\
\hline IC $95 \%$ para a média & $8,7 \pm 1,23$ & Mín-Max: 4-12 \\
\hline \multicolumn{3}{|l|}{ Cor } \\
\hline Negra & 9 & 45,0 \\
\hline Branca & 11 & 55,0 \\
\hline IC $95 \%$ para branca & & \\
\hline \multicolumn{3}{|l|}{ Escolaridade } \\
\hline$<$ Fundamental & 7 & 35,0 \\
\hline Fundamental & 13 & 65,0 \\
\hline \multicolumn{3}{|c|}{$\begin{array}{l}\text { Renda per capita familiar } \\
\text { (em salários mínimos) }\end{array}$} \\
\hline$<0,5$ & 6 & 30,00 \\
\hline 0,5 a 1,0 & 9 & 45,00 \\
\hline$>1,0$ & 5 & 25,00 \\
\hline Média (DP) & $0,88(0,70)$ & Mediana: 0,75 \\
\hline IC $95 \%$ para a média & $0,88 \pm 0,33$ & Min-Max: 0,18-3,25 \\
\hline
\end{tabular}

escolaridade, 13 crianças (65\%) estavam no nível fundamental, e a renda média per capita era inferior a um salário mínimo $(0,88$ e DP $=0,70)$.

A Tabela 2 mostra que as malformações congênitas representam $60 \%$ (12) das causas das ostomias; predominando as urostomias de caráter provisório (11 ou 55\%), com tempo médio de 53,65 meses ( $\mathrm{DP}=60,59)$ de confecção e com período de pós-operatório inferior a 50 meses para a maioria das crianças (13 ou 65\%).

\section{Análise da QV das crianças ostomizadas}

Os escores totais e parciais sobre a QV das crianças ostomizadas, a partir da aplicação da EQVC junto às crianças e mães, são apresentados na Tabela 3.

De uma variação possível de 0 a 78, os escores médios totais da EQVC foram 51,95 (DP=7,90) e 49,60 (DP=5,60), respectivamente, na ótica das crianças (EQVCr) e mães (EQVmãe), sem diferença estatisticamente significativa entre eles $(\mathrm{p}<0,05)$. Os fatores lazer, família e funções obtiveram, nesta ordem, os maiores valores $(8,29 \mathrm{e} \mathrm{DP}=0,92$; 11,41 e $\mathrm{DP}=0,17 ; 10,27$ e $\mathrm{DP}=0,11$, respectivamente), respeitando-se as variações possíveis. Ao ser considerada a nota de corte 48 , verificou-se que a maioria dos índices (70\%) encontra-se na faixa igual e acima de 48 , para ambos grupos, indicando QV positiva.

Tabela 2 - Distribuição das variáveis clínicas da amostra - São Paulo, 2001

\begin{tabular}{lcc}
\hline Estoma & \multicolumn{2}{c}{ Distribuição } \\
& $\mathbf{N}^{\mathbf{0}}$ & $\%$ \\
\hline Causa & & \\
$\quad$ Malformação congênita & 12 & 60,0 \\
Trauma & 4 & 20,0 \\
Doença inflamatória & 2 & 10,0 \\
Neoplasia & 2 & 10,0 \\
Tipo & & \\
Colostomia & 6 & 30,0 \\
Ileostomia & 2 & 10,0 \\
Urostomia & 11 & 55,0 \\
Colostomia E e urostomia & 1 & 5,0 \\
Caráter & & \\
Definitivo & 9 & 45,0 \\
Provisório & 11 & 55,0 \\
IC 95\% para provisório & $55,0 \pm 23,88$ & \\
Tempo (em meses) & & 65,0 \\
$\quad$ < 50 & 13 & 10,0 \\
50 a 100 & 2 & 25,0 \\
$>100$ & 5 & Mediana: 24,5 \\
Média (DP) & $53,65(60,59)$ & Min-Max: $2,0-168,0$ \\
IC 95\% para a média & $53,65 \pm 28,36$ & \\
&
\end{tabular}


Tabela 3 - Medidas descritivas dos escores de QV e seus fatores - São Paulo, 2001

\begin{tabular}{|c|c|c|c|c|c|c|c|}
\hline \multirow[t]{2}{*}{ Variável } & \multirow[t]{2}{*}{$\mathbf{N}^{\mathbf{o}}$} & \multirow[t]{2}{*}{ Média } & \multirow[t]{2}{*}{ DP } & \multicolumn{2}{|c|}{ Variação } & \multirow[t]{2}{*}{ Mediana } & \multirow[t]{2}{*}{ IC $95 \%$} \\
\hline & & & & Obtida & Possível & & \\
\hline Autonomia & 14 & 8,14 & 1,70 & 4 a 11 & 0 a 15 & 8,50 & $8,14 \pm 0,98$ \\
\hline Lazer & 17 & 8,29 & 0,92 & 6 a 9 & 0 a 9 & 9,00 & $8,29 \pm 0,47$ \\
\hline Funções & 18 & 10,27 & 0,11 & 7 a 13 & 0 a 15 & 11,00 & $10,27 \pm 0,88$ \\
\hline Família & 17 & 11,41 & 0,17 & 6 a 15 & 0 a 15 & 13,00 & $11.41 \pm 1,49$ \\
\hline EQVCr & 20 & 51,95 & 7,90 & 39 a 66 & 0 a 78 & 52,50 & $51,95 \pm 3,70$ \\
\hline EQVmãe & 20 & 49,60 & 5,60 & 38 a 63 & 0 a 78 & 50,00 & $49,60 \pm 2,62$ \\
\hline
\end{tabular}

Analisando-se os escores médios de cada item componente da escala, de forma independente, verificou-se que "longe da família" e "hospitalização" obtiveram os menores valores para crianças e mães; enquanto "aniversário", "avós" e "férias" alcançaram os maiores valores para as crianças, diferentemente de suas mães.

No teste de correlações bisseriais por ponto, constatouse que somente a escolaridade mostrou correlação negativa e estatisticamente significativa com o EQVCr total $(r=-$ 0,63 e p $<0,05$ ), sugerindo que crianças com menor escolaridade apresentam os menores escores de QV. Esse resultado é corroborado no teste de comparação entre os grupos de crianças com maior e menor escolaridade, quando o escore total de QV das crianças com maior escolaridade $(55,54)$ supera, com significância estatística ( $\mathrm{p}=0,003)$, aquele encontrado para crianças com escolaridade abaixo da fundamental $(45,28)$.

Além disso, detectaram-se correlações estatisticamente significativas, negativas, entre o EQVmãe e a escolaridade da criança $(r=-0,56)$, e positivas, entre o EQVmãe e os fatores família $(r=0,86)$, autonomia $(r=0,60)$ e funções $(r$ $=0,59)$ apontadas pelas crianças além do $\mathrm{EQVCr}(\mathrm{r}=0,82)$. Esses resultados sugerem que as mães percebem pior QV entre as crianças com menor nível de escolaridade e melhor QV relacionada aos aspectos inerentes à família, à autonomia e às funções, e também aos maiores escores de QV na ótica das crianças.

\section{Discussão}

Pensar em qualidade de vida leva a refletir sobre tudo aquilo que se relaciona com o grau de satisfação, felicidade e bem-estar, embora não exista, atualmente, consenso sobre o seu significado. Em se tratando de crianças, torna-se mais difícil ainda partilhar de uma concepção, visto que qualidade de vida na infância está relacionada, principalmente, a brincadeiras, harmonia e prazer, e varia conforme as fases do crescimento, o desenvolvimento infantil e as relações familiares. Além disso, tradicionalmente, a QV das crianças têm sido verificada através das percepções dos pais ou responsáveis. Esse panorama tem-se modificado, em parte, por meio do desenvolvimento de alguns instrumentos específicos de medida, destinados à população pediátrica, dentre os quais o AUQUEI ou EQVC, já adaptado transculturalmente para o Brasil.

Reconhecendo-se que as situações que alteram a saúde da criança, ou que deixam seqüelas, como as ostomias, impõem questionamentos acerca do comprometimento da QV no que tange aos mecanismos de enfrentamento e adaptação diante das limitações físicas ou comportamentais ${ }^{13,20-23}$ e a possibilidade de utilização da EQVC motivaram a realização deste estudo, envolvendo crianças ostomizadas, cuja literatura é absolutamente escassa em nível internacional e ausente em nosso meio.

As 20 crianças incluídas neste estudo, 10 meninos e 10 meninas, com idade entre 4 e 12 anos, atendidas em serviço de referência para a criança ostomizada na cidade do estado de São Paulo, eram portadoras, principalmente, de estomas urinários temporários, oriundos de malformações congênitas, como esperado.

Quanto à QV das crianças ostomizadas, propriamente dita, inicialmente procedemos à validação da $\mathrm{EQVC}$, analisando sua confiabilidade e validades externa e de construto, que se mostraram satisfatórias para este estudo. Estabelecidas as propriedades psicométricas da EQVC para avaliar a QV das crianças ostomizadas, obtiveram-se escores totais médios de 51,95 (DP = 7,90) e 49,60 ( $\mathrm{DP}=5,60)$, respectivamente, para as crianças e suas mães, positiva e fortemente correlacionados entre si $(r=0,82)$. Esses resultados, que denotam percepções positivas sobre a QV das crianças ostomizadas $(\geq 48)$, tanto por elas como por suas mães, assemelham-se aos encontrados por Assumpção Jr. et al. ${ }^{13}$ junto a crianças saudáveis, e contrariam as projeções 
desses autores, de que esses índices são pouco correlacionados, quando se trata de crianças doentes ou hospitalizadas.

Ao serem examinados os fatores componentes do EQVCr, o lazer obteve o maior escore na ótica das crianças, relacionado principalmente a "aniversário", "avós" e "féri$a s$ ", que também alcançaram os maiores índices por item, revelando a importância desses aspectos - brincar e lazer para a sensação subjetiva de bem-estar, não só para as crianças saudáveis ${ }^{13-15}$, como também para crianças hospitalizadas, ou com alterações crônicas ${ }^{20}$, como as ostomias.

Além do lazer, a família foi percebida como fonte de bem-estar para a QV das crianças não só por elas mesmas, ao obter o segundo escore mais elevado, mas também por suas mães $(r=0,86)$, ao ser considerada componente fundamental para a construção de um autoconceito positivo e um nível satisfatório de auto-estima ${ }^{5-6}$, apesar da presença da ostomia.

Por outro lado, a autonomia mostrou-se como o fator mais comprometido na percepção das crianças, com o menor índice comparativamente aos demais, diferentemente das mães que consideraram a autonomia como um dos aspectos inerentes à maior $\mathrm{QV}$ das crianças $(\mathrm{r}=0,60)$. Esse fator incluiu o item que obteve o menor valor tanto entre as crianças como entre as mães, qual seja "longe da família". Deve-se ressaltar que, tanto na etapa pré-escolar como na escolar, a busca de independência e autonomia na primeira, e o desenvolvimento de competências e habilidades, na segunda ${ }^{4}$, podem estar comprometidos pela presença da ostomia $^{7}$, e todas as repercussões que ela envolve, anteriores à sua realização - com a doença e hospitalização - e conseqüentes à sua presença. Nesse sentido, a hospitalização também se destaca como importante domínio para a percepção de bem-estar das crianças, tanto para elas como para suas mães, ao mostrar baixos valores em ambos os grupos.

Outro aspecto coincidente esteve relacionado à percepção de piores níveis de bem-estar e QV para crianças com menor escolaridade, em ambos grupos, talvez associado às maiores demanda e dependência em relação à aprendizagem de habilidades mais complexas para aquisição e desenvolvimento do autocuidado.

Ressalta-se que este estudo, apesar de casuística reduzida - condicionada principalmente pela faixa etária em que o instrumento pode ser aplicado - é inédito e traz à tona importantes resultados para a prática clínica. A constatação das percepções semelhantes entre crianças e mães a respeito de aspectos fundamentais para o maior nível de bemestar, como a família, o lazer e a autonomia, e a QV como um todo, podem repercutir na adequação de modelos de cuidar em estomaterapia, que valorizem ou priorizem tais componentes da QV das crianças ostomizadas, assim como para o desenvolvimento de políticas de atendimento diretamente voltadas a essa clientela específica.

\section{Agradecimento}

Gostaríamos de agradecer à colega enfermeira estomaterapeuta Donata Maria de Souza, responsável pelo Serviço de Referência, pela colaboração no processo de localização e agendamento prévio das crianças com ostomias.

\section{Referências bibliográficas}

1. Rocha RFC. Estomas na criança. In: Santos VLCG, Cesaretti IUR, editores. Assistência em Estomaterapia: cuidando do ostomizado. $1^{\text {a }}$ ed. São Paulo: Ateneu; 2000.p.69-81.

2. Bóscollo ACP, Stopa MJR, Vicente YAMVA. Peculiaridades dos estomas em crianças. In: Crema E, Silva R. Estomas: uma abordagem interdisciplinar. $1^{\text {a }}$ ed. Uberaba: Editora Pinti; 1997. p.107-24.

3. Carvalho WAF, Yamamoto MS, Del Cistia MEGF. A criança ostomizada. In: Santos VLCG, Cesaretti IUR, editores. Assistência em Estomaterapia: cuidando do ostomizado. $1^{\mathrm{a}}$ ed. São Paulo: Ateneu; 2000.p.133-54.

4. Neumann E. A Criança. $1^{a}$ ed. São Paulo: Editora Cultrix; 1980.

5. Mussen PH. O desenvolvimento psicológico da criança. $9^{\mathrm{a}}$ ed. Rio de Janeiro: Editora Zahar; 1978.

6. Goldman R. Cross cultural adaptation of a program to evolve parents in there children's learning. Child Welfare 1973;52:521-9.

7. Lalande FL. Ostomies in childhood: a challenge for the enterostomal therapy nurse. Proceedings of the XI Biennial Congress of the World Council of Enterostomal Therapists; 1996; Jerusalém, Israel. Jerusalém: WCET; 1996.p.60-61.

8. Hardin D. The school age child and the school nurse. Am J Nurs 1974;74:1476-8.

9. Waechter H. Enfermagem Pediátrica. $9^{\mathrm{a}}$ ed. Rio de Janeiro: Editora Interamericana; 1978.

10. Farquhar M. Definitions of quality of life: a taxonomy. J Adv Nurs 1995;22:502-508.

11. Santos VLCG, Kimura M. Qualidade de vida e reabilitação do ostomizado. In: Santos VLCG, Cesaretti IUR, editores. Assistência em Estomaterapia: cuidando do ostomizado. $1^{\mathrm{a}} \mathrm{ed}$. São Paulo: Ateneu; 2000.p.453-75.

12. Bowling A. Measuring health - a review of Quality of Life measurement scales. $2^{\mathrm{a}}$ ed. Philadelphia: Open University Press; 1997.

13. Assumpção FR Jr, Kuzynski E, Sprovieri MH, Aranha EMG. Escala de avaliação da qualidade de vida (Autoquestionnaire qualité de vie enfant imagé): validade e confiabilidade de uma escala para qualidade de vida em crianças de 4 a 14 anos. Arq Neuropsiquiatr 2000;58:7-15.

15. Guyatt GH, Feeny DH, Patrick DL. Measuring health-related quality of life. Ann Intern Med 1993;118:622-629.

16. Manificat S, Dazord A, Cochat P, Nicolas J. Evaluation de la vie en pediatric comment recuellir le point de vie de l'enfant. Arch Pediatric1997;4:1238-46.

17. Jeter K. The pediatric patient. In: Broadwell D, Jackson B, editores. Principles of ostomy care. St. Louis: CV Mosby; 1982. p.489-533.

18. Erwin-Toth P. Teaching ostomy care to the pediatric client: a developmental approach. Journal of Enterostomal Therapy 1988;15:126-30.

19. Erwin-Toth P. The holes within the whole: childhood ostomy surgery - a development view. Proceedings of the XI Biennial Congress of the World Council of Enterostomal Therapists; 1996; Jerusalém, Israel. Jerusalém: WCET;1996:53-5. 
20. Erwin-Toth P. The effect of ostomy surgery between the ages of 6 and 12 years on psychosocial development during childhood, adolescence and young adulthood. WOCN J 1999;26:77-85.

21. Hartnick CJ, Giambra BK, Bissel C, Fitton CM, Cotton RT, Parsons SK. Final validation of the Pediatric Tracheotomy Health Status Instrument (PTHSI). Otolaryngol Head Neck Surg 2002;126:228-33.

22. Manificat S, Dazord A. Évaluation de la qualité de vie de l'enfant: validation d'un questionnaire, premiers résultats. Neuropychhiatric Enfance Adolesc 1997;45:6-114.

23. Souza LJEX, Barroso MGT. Qualidade de vida na criança acidentada. Rev Texto Contexto 1999;8:308-17.
24. Rosenfeld RM, Bhaya MH, Bower CM, Brookhouser PE, Casselbrant ML, Chan KH, et al. Impact of tympanostomy tubes on child quality of life. Arch Otolaryngol Head Neck Surg 2000;126:585-92.

Endereço para correspondência:

Dra. Vera Lúcia Conceição de Gouveia Santos

Escola de Enfermagem da USP

Av. Dr. Enéas de Carvalho Aguiar, 419 - Cerqueira César CEP 05403-000 - São Paulo, SP

Fone: (11) 3066.7565 / 3066.7544 - Fax: (11) 3066.7546

E-mail: veras@usp.br 